\title{
Modélisation des mises en suspension des sédiments de fond dans le détroit du Pas-de-Calais en condition de tempête - comparaison avec les observations
}

\author{
Nicolas GUILLOU ${ }^{1}$, Georges CHAPALAIN ${ }^{2}$ \\ Centre d'Etudes Techniques Maritimes et Fluviales, \\ Laboratoire Génie Côtier et Environnement, Technopôle Brest-Iroise, \\ BP 5, 29280 Plouzané, France. \\ ${ }^{1}$ Ingénieur de recherche, nicolas.guillou@developpement-durable.gouv.fr \\ ${ }^{2}$ Directeur du LGCE, georges.chapalain@developpement-durable.gouv.fr
}

\section{Résumé :}

Un modèle hydro-sédimentaire tridimensionnel multiclasse basé sur le couplage des codes de circulation COHERENS et de propagation de houle SWAN est appliqué à l'environnement du détroit du Pas-de-Calais et des zones côtières adjacentes au cours d'une tempête. Le modèle reproduit les mesures de houle, des courants de marée, de la vitesse de frottement liée au courant et de la concentration de sédiment en suspension totale au large de la plage de Merlimont. Les effets de la houle sur ces paramètres et les concentrations des diverses classes sédimentaires en suspension sont mis en évidence.

\begin{abstract}
:
A three-dimensional modelling of polydispersive suspended sediment transport based on the coupling of the circulation model COHERENS and the wave propagation model SWAN is applied in the Dover Strait and its coastal adjacent areas during a storm. The model reproduces measurements of waves parameters, tidal currents, bottom tidal shear stress velocity and the total suspended sediment concentration off Merlimont beach. The wave effects on these parameters and the concentration of the different classes of suspended sedimentary particles are highlighted.
\end{abstract}

\section{Mots-clés :}

modélisation, transport sédimentaire multiclasse, suspension, interaction houlecourant en couche limite de fond. 


\section{Introduction}

Le domaine côtier de la Manche est caractérisé par une forte variabilité des composantes hydrodynamiques de marée (e.g., SALOMON \& BRETON, 1991) et une hétérogénéité des sédiments de fond (e.g., VASLET et al., 1978: LARSONNEUR et al., 1982) qui influent sur la composition et la répartition spatiale des mises en suspension (e.g., GUILLOU, 2007). A l'échelle locale des zones côtières, les faibles profondeurs favorisent également l'action de la houle sur le mouvement des sédiments de fond (GROCHOWSKI \& COLLINS, 1994). Cependant, le nombre de modèles numériques consacrés en Manche aux effets de la houle sur le transport sédimentaire reste limité (e.g., VELEGRAKIS et al., 1996 ; CUGIER, 2000 ; VILLARET \& DAVIES, 2004 ; GUILLOU, 2007). La présente étude vise à analyser à l'aide de codes numériques les effets d'une houle de tempête superposée au courant de marée sur les mises en suspension de particules sédimentaires de fond de différentes tailles. Ce travail se situe dans le prolongement des comparaisons modèles-mesures effectuées le long du littoral méridional du détroit du Pas-de-Calais en condition de marée seule par GUILLOU \& CHAPALAIN (2006). L'article s'organise selon les étapes suivantes. Après une description succincte des codes numériques et des conditions de modélisation (Section 2), les résultats des simulations sont comparés aux mesures in situ effectuées au large de la plage de Merlimont et discuter pour mettre en évidence les effets de la houle par rapport à une situation de temps calme (Section 3). Enfin, la section 4 synthétise ces résultats.

\section{Modèles numériques et conditions de simulation}

\subsection{Modèles numériques}

Le modèle hydro-sédimentaire est basé sur le code de circulation tridimensionnelle COHERENS (LUYTEN et al., 1999) adapté à la résolution de l'advection et de la dispersion de particules sédimentaires non-cohésives de différentes tailles (GUILLOU, 2007). Il est couplé au code de propagation spectrale de houle SWAN (BOOIJ et al., 1999) via un module d'interaction houlecourant en couche limite benthique basé sur la théorie de GRANT \& MADSEN (1979) dans lequel

i. les modifications sur le profil vertical du coefficient de diffusion turbulente $\lambda_{\mathrm{T}}$ sont imposées au niveau d'un sous-maillage de la demimaille de fond servant à un calcul optimisé du flux de dépôt des particules sédimentaires à l'interface eau-sédiment,

ii. la contrainte de cisaillement totale $\tau_{\mathrm{cw}}$ est calculée à partir de la solution analytique de l'équation du mouvement dans la couche limite de houle (GRANT \& MADSEN, 1979), 
iii. la contrainte de cisaillement liée au courant $\tau_{\mathrm{c}}$ et la vitesse de frottement associée $\mathrm{u}_{*_{\mathrm{c}}}$ prennent en compte les effets de rugosité apparente ressentie par le courant au-dessus de la couche limite de houle.

Des détails supplémentaires sur les développements entrepris sur les modules d'interaction houle-courant et de transport sédimentaire sont disponibles dans GUILLOU (2007).

\subsection{Conditions de simulation}

Le modèle hydrosédimentaire est implanté sur deux zones gigognes analogues à celles utilisées pour les prédictions en condition de marée seule (GUILLOU \& CHAPALAIN, 2006) : (i) un domaine \#1 couvrant la quasi-totalité de la Manche et la partie méridionale de la Mer du Nord avec une résolution de $2 \mathrm{~km}$ et (ii) un domaine \#2 correspondant au détroit du Pas-de-Calais avec un maillage de $500 \mathrm{~m}$ de résolution (Fig. 1).

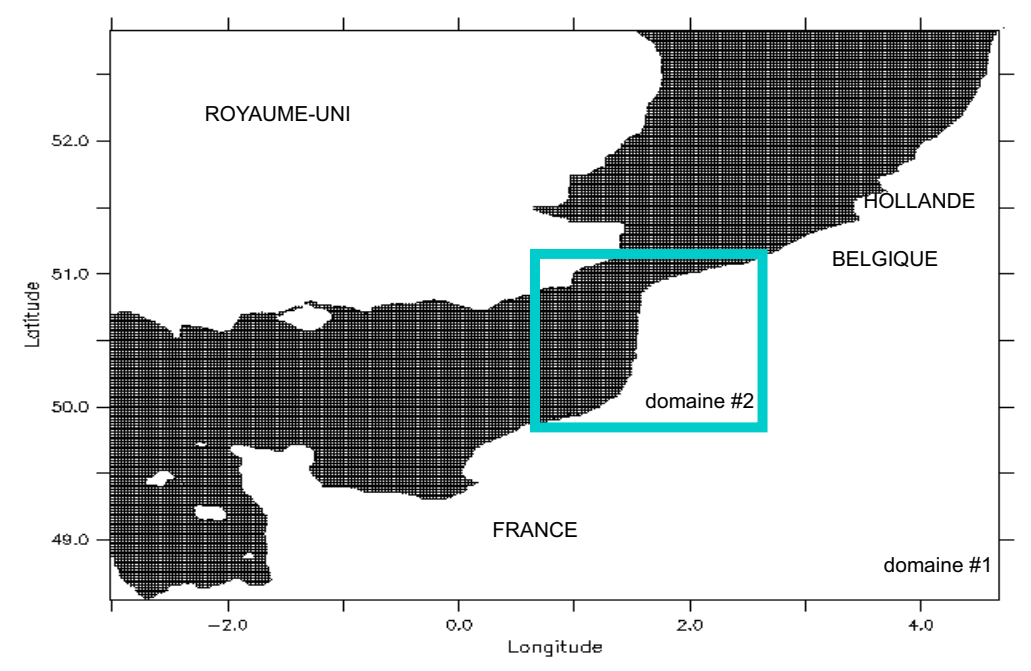

Figure 1 : Domaines de calcul \#1 et \#2.

Sur le domaine \#1, le paramètre de rugosité est fixé à $\mathrm{z}_{0}=0,0035 \mathrm{~m}$ de la même manière que LUYTEN et al. (1999) en Mer du Nord. Sur le domaine \#2, les rugosités de grain et de forme liées aux rides de courant sont prises en compte par l'application d'une technique originale d'interpolation des distributions granulométriques des échantillons de sédiment de fond aux différents noeuds du maillage de calcul (LEPRETRE et al., 2006). Cette technique est appliquée aux 11 classes granulométriques, s'étalant des silts $(\mathrm{d}<50 \mu \mathrm{m})$ aux cailloutis $(\mathrm{d}>2 \mathrm{~cm})$ et affleurements rocheux, issues des données des campagnes « RCP 378 Benthos de la Manche » (e.g., CABIOCH et al., 1977) (Fig. 2).

L'élévation de la surface libre est imposée aux limites ouvertes du domaine \#1 à 
partir des résultats d'un modèle bidimensionnel horizontal appliqué au domaine épicontinental des îles britanniques (José OZER - communication personnelle). Les conditions aux frontières du domaine \#2 sont la surface libre et les courants prédits sur le domaine \#1.

Les transports sédimentaires sont uniquement calculés sur le sous-domaine \#2 ou la répartition spatiale des distributions granulométriques est effectivement prise en considération.

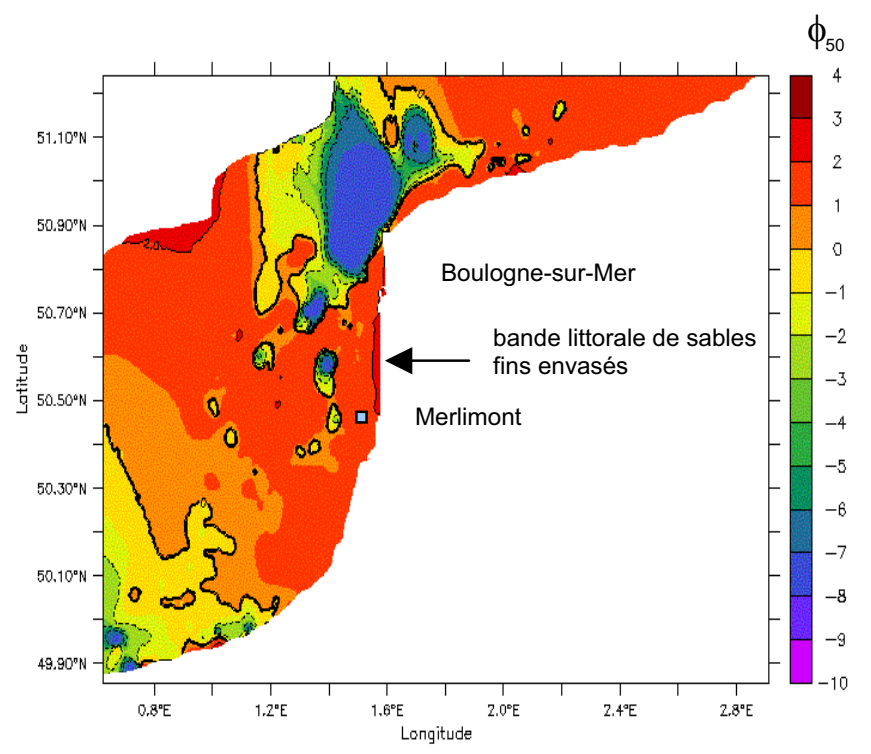

Figure 2: Répartition spatiale du diamètre médian $\phi_{50}=-\log _{2}\left(d_{50}\right)$, avec $d_{50}$ en $\mathrm{mm}$, sur le domaine \#2 calculée par la technique proposée par LEPRETRE et al. (2006).

Le modèle de propagation de houle SWAN est utilisé sur le maillage du domaine \# 2 en mode instationnaire en intègrant (i) les variations de la hauteur d'eau et des courants de marée prédits par COHERENS et (ii) les phénomènes de génération et de dissipation par le vent considéré spatialement uniforme et dont la chronique est la mesure effectuée au sémaphore de Boulogne-sur-Mer. Les conditions limites de houle correspondent à un spectre de "JONSWAP" imposé uniformément le long des frontières Ouest et Nord à partir des données de l'Atlas numérique de houle le long des côtes françaises (BENOIT \& LAFON, 2004).

\section{Applications}

Les résultats du modèle sont comparés, en octobre 1998 au cours d'un évènement de houle associé à un coup de vent de Nord, aux mesures effectuées par la Station d'Acquisition de Mesures Benthique Autonome (SAMBA) au niveau d'un site littoral situé au large de la plage de Merlimont (longitude : $1^{\circ} 537 \mathrm{E}$, latitude : $50^{\circ} 450 \mathrm{~N}, \mathrm{~d}_{50}=256 \mu \mathrm{m}, 13,5 \mathrm{~m}$ de niveau moyen) (Fig. 2) à $0,3,0,6,0,9$ et $1,4 \mathrm{~m}$ 
au-dessus du fond. Ce site de mesure est notamment caractérisé par la présence d'une bande littorale de sables fins envasés au Nord. Des détails supplémentaires sont disponibles dans CHAPALAIN \& THAIS (2000).

\subsection{Houle}

La Figure 3 présente les prédictions de la hauteur $\mathrm{h}_{\mathrm{m} 0}$, de la direction, de la période de pic et de la vitesse orbitale $u_{\mathrm{b}, \text { rms }}$ comparées aux mesures effectuées à partir du capteur de pression positionné sur la SAMBA.
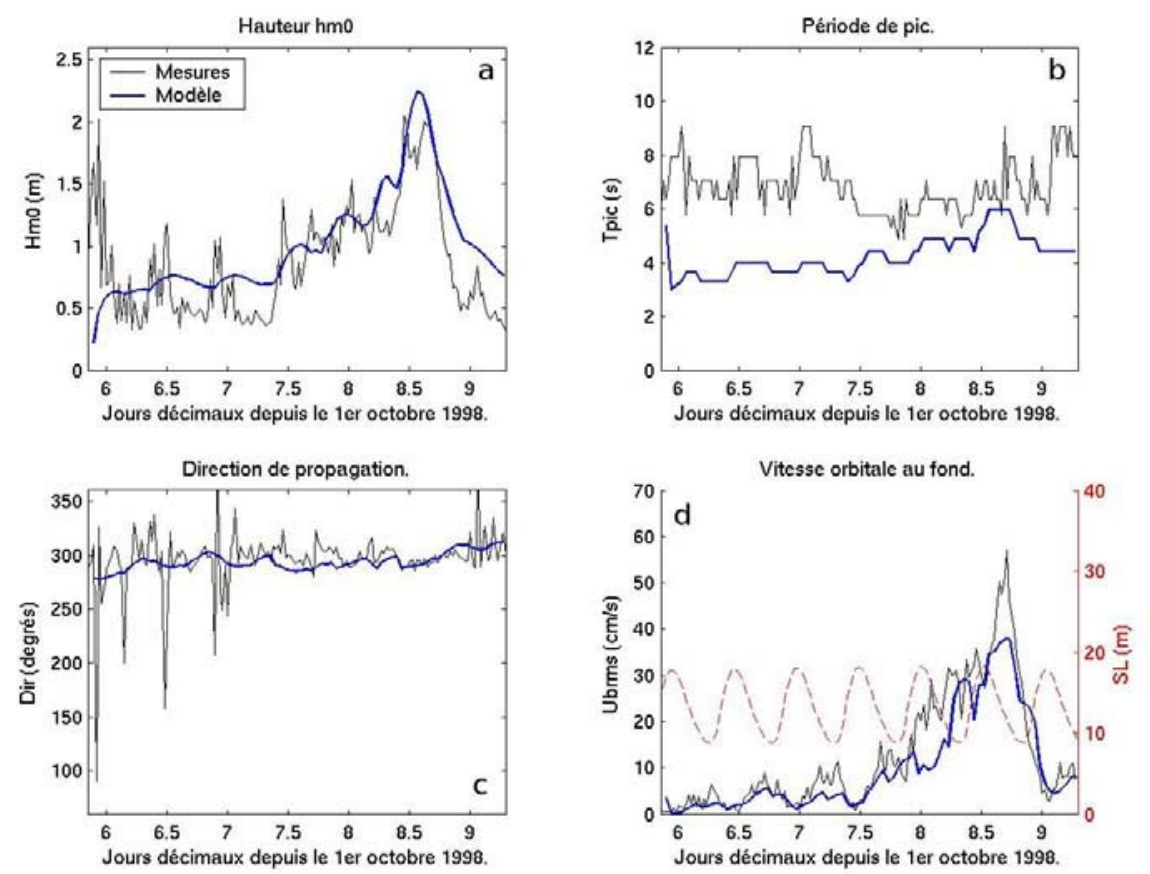

Figure 3 : Chroniques de la hauteur $h_{m 0}(a)$, de la période de pic (b), de la direction de propagation repérée par rapport à la longitude dans le sens trigonométrique (c), de la vitesse orbitale $u_{b, r m s}(d)$, mesurées et prédites, et de la hauteur d'eau mesurée (d) au site de Merlimont en octobre 1998.

L'évolution de la hauteur $\mathrm{h}_{\mathrm{m} 0}$ (Fig. 3-a) est reproduite correctement avec un écart inférieur à $12 \%$ au pic de tempête, le 8 octobre 1998. Le modèle approche également les phénomènes de gonflement de la houle par les courants de flot en phase avec la marée haute et opposés au sens de propagation de la houle en provenance du Nord (Fig. 3-c). Les prédictions reproduisent les variations de la vitesse orbitale $u_{b, r m s}$ (Fig. 3-e) avec (i) par temps calme des pics d'amplitude en phase avec les basses-mers et (ii) au pic de tempête du 8 octobre 1998 une augmentation d'un facteur 6 de $u_{b, r m s}$. La vitesse orbitale est cependant sous- 
estimée au pic de tempête, cette différence résultant en partie des écarts obtenus sur la période (Fig. 3-b) attribués à l'utilisation d'un fetch réduit pour nos simulations.

\subsection{Paramètres hydrodynamiques}

Les prédictions des courants à 0,3 et $0,6 \mathrm{~m}$ au-dessus du fond (Fig. 4) s'accordent avec les mesures avec (i) par temps calme $\left(\mathrm{h}_{\mathrm{m} 0}<0,8 \mathrm{~m}\right)$, un écart moyen prédictions-observations de l'amplitude des courants inférieur à $10 \%$, et (ii) durant la tempête $\left(\mathrm{h}_{\mathrm{m} 0}>1,2 \mathrm{~m}\right)$, un écart moyen de $15 \%$. Au pic de tempête du 8 octobre 1998, la houle, de part l'augmentation du paramètre de rugosité apparente de $4,5 \mathrm{~mm}$, conduit à une atténuation de $24 \%$ de l'amplitude des courants.

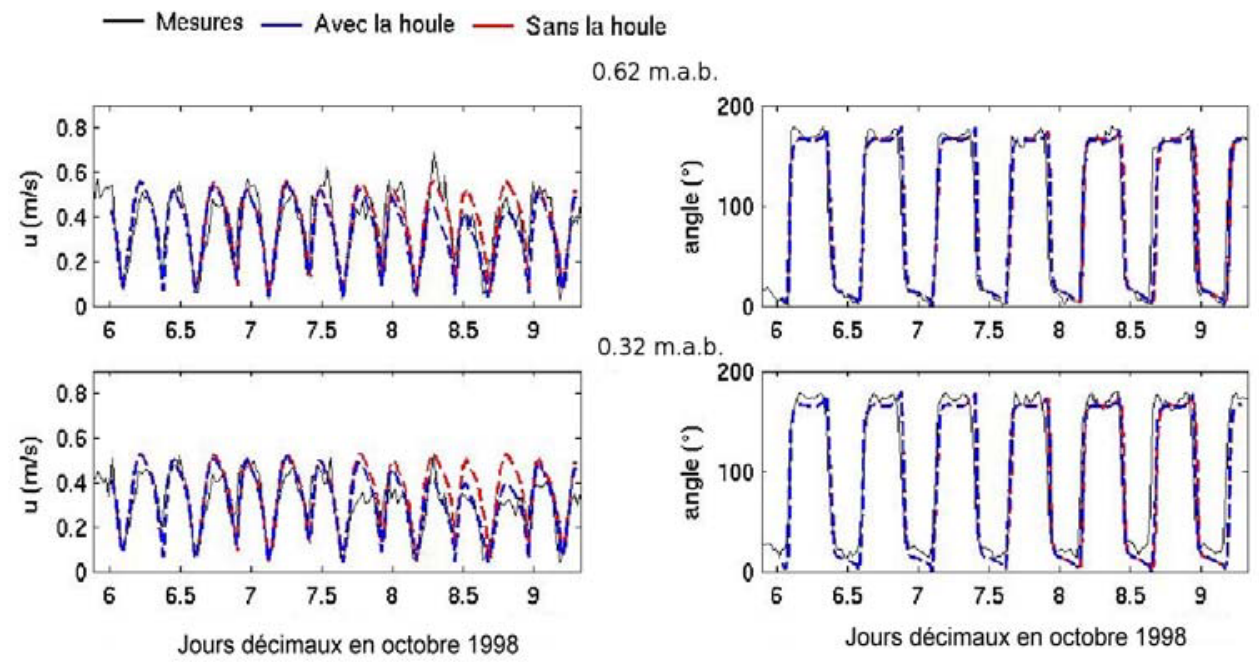

Figure 4: Chroniques du module et de la direction de la vitesse repérée par rapport au Nord dans le sens horaire, mesurée (ligne continue) et prédites (ligne en pointillés) sans et avec prise en compte des conditions de houle, à 0,3 et 0,6 $\mathrm{m}$ au-dessus du fond au site de Merlimont en octobre 1998.

La Figure 5 présente les prédictions de la vitesse de frottement due au courant $\mathrm{u} *_{\mathrm{c}}$ comparées aux mesures. L'écart avec les mesures est en moyenne de $15 \%$. L'augmentation du frottement apparent se traduit par une augmentation sensible, de $5 \%$, de la vitesse de frottement liée au courant. Au pic de tempête, $\mathrm{u} *_{\mathrm{c}}$ passe ainsi de $4,37 \mathrm{~cm} . \mathrm{s}^{-1}$ en condition de marée seule à $4,55 \mathrm{~cm} \cdot \mathrm{s}^{-1}$ dans le cas d'une houle superposée à un courant de marée. Cette faible évolution résulte d'une compétition entre les forçages exercés par les courants de marée et la houle. 


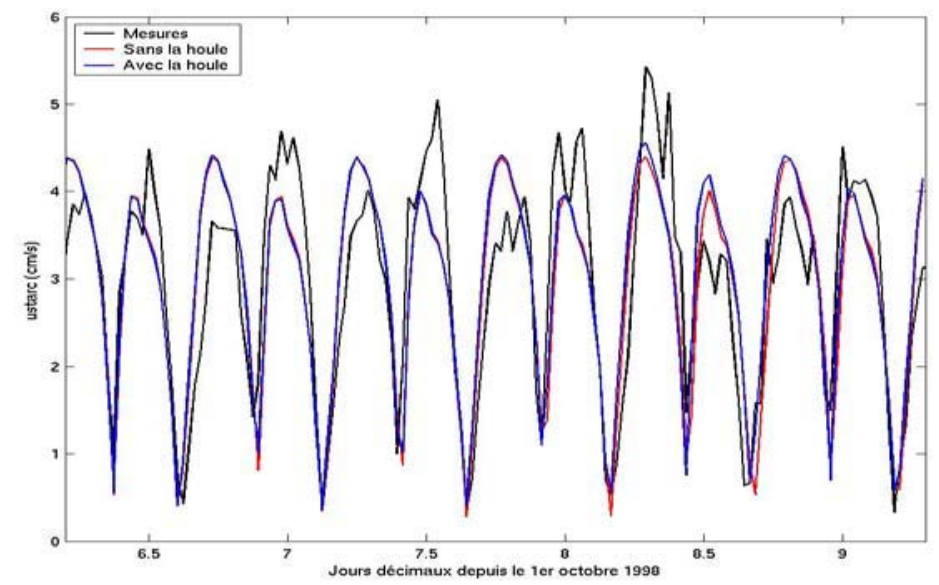

Figure 5 : Chroniques de la vitesse de frottement due au courant $u *_{c}$ mesurée et prédites sans et avec prise en compte des conditions de houle au site de Merlimont en octobre 1998.

\subsection{Concentration de sédiment en suspension}

Les prédictions de la concentration de sédiment en suspension (CSS) totale sont comparées aux mesures effectuées à $0,3 \mathrm{~m}$ au-dessus du fond (Fig. 6). Par temps calme, le modèle reproduit les pics de CSS particulièrement marqués en jusant, lorsque la vitesse orbitale $u_{b, r m s}$ est maximale (Fig. 3-d), avec un écart prédictionsobservations qui se réduit progressivement jusqu'à atteindre $5 \%$ au deuxième pic de jusant du 7 octobre 1998. Durant la tempête, le modèle représente correctement l'amplitude du premier pic de CSS du 8 octobre 1998, mais sous-estime la CSS totale atteinte au pic de tempête en raison de la difficulté à approcher $u_{b, r m s}$ à cet instant (Section 3.1). Ces simulations montrent que la houle accroît d'un facteur 4 les mises en suspension à $0,3 \mathrm{~m}$ au-dessus du fond par rapport à une situation de temps calme. Cette augmentation résulte d'une exacerbation de la contrainte de cisaillement superficielle passant de 2,6 N.m à 7,4 N.m ${ }^{-2}$. Enfin, on peut noter la présence de pics de CSS en flot non identifiés au niveau des mesures, en particulier dans l'après-midi du 8 octobre 1998.

Le signal de CSS totale prédit résulte des mises en suspension des quatre premières classes (Fig. 7) avec

(i) une contribution majoritaire des sables fins $\left(d_{3}=150 \mu \mathrm{m}\right)$ en suspension dont la CSS atteint $200 \mathrm{mg} .1^{-1}$ au pic de tempête,

(ii) une contribution secondaire des silts $\left(\mathrm{d}_{1}=25 \mu \mathrm{m}\right)$ et des sables très fins $\left(\mathrm{d}_{2}=75 \mu \mathrm{m}\right)$ dont l'évolution est semi-diurne avec des maxima de CSS en 
fin de jusant,

(iii) et une contribution minoritaire des sables moyens $\left(d_{4}=350 \mu \mathrm{m}\right)$ mis uniquement en suspension au cours de la tempête.

L'analyse synoptique des champs de CSS autour du site de mesure mets en évidence des mises en suspension locales des sables fins à moyens (classes 3 et 4) superposées à la concentration des sédiments fins (classes 1 et 2) advectés en période de jusant de la bande littorale de sables fins envasés au Nord du site de mesure (Fig. 2).

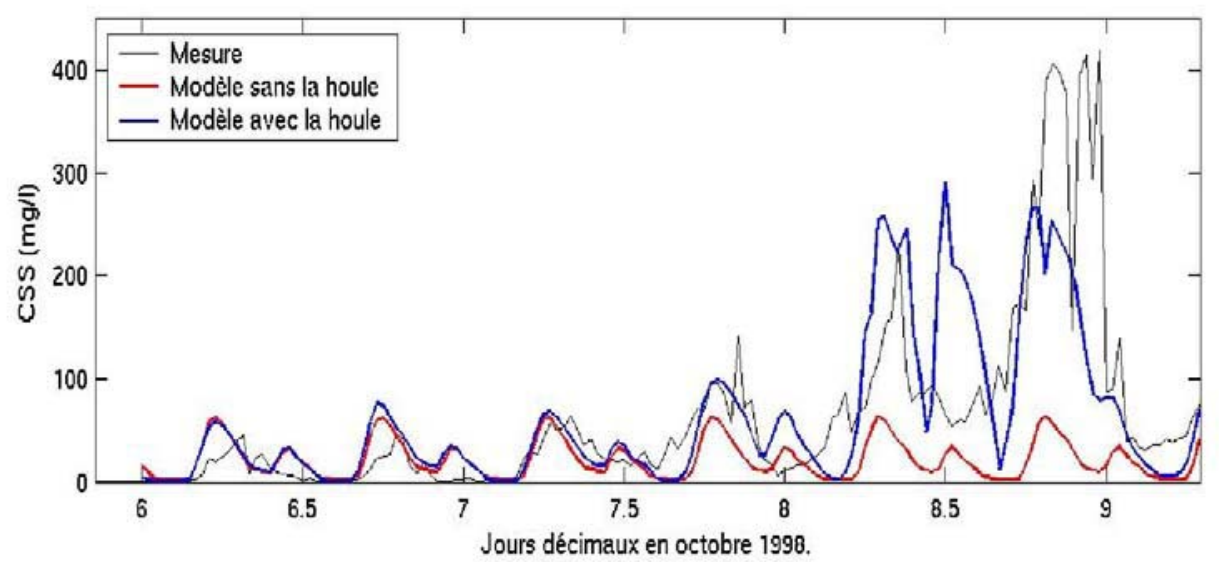

Figure 6: Chroniques de la CSS totale mesurée et prédites sans et avec prise en compte des conditions houle, à 0,3 m au-dessus du fond au site de Merlimont en octobre 1998.

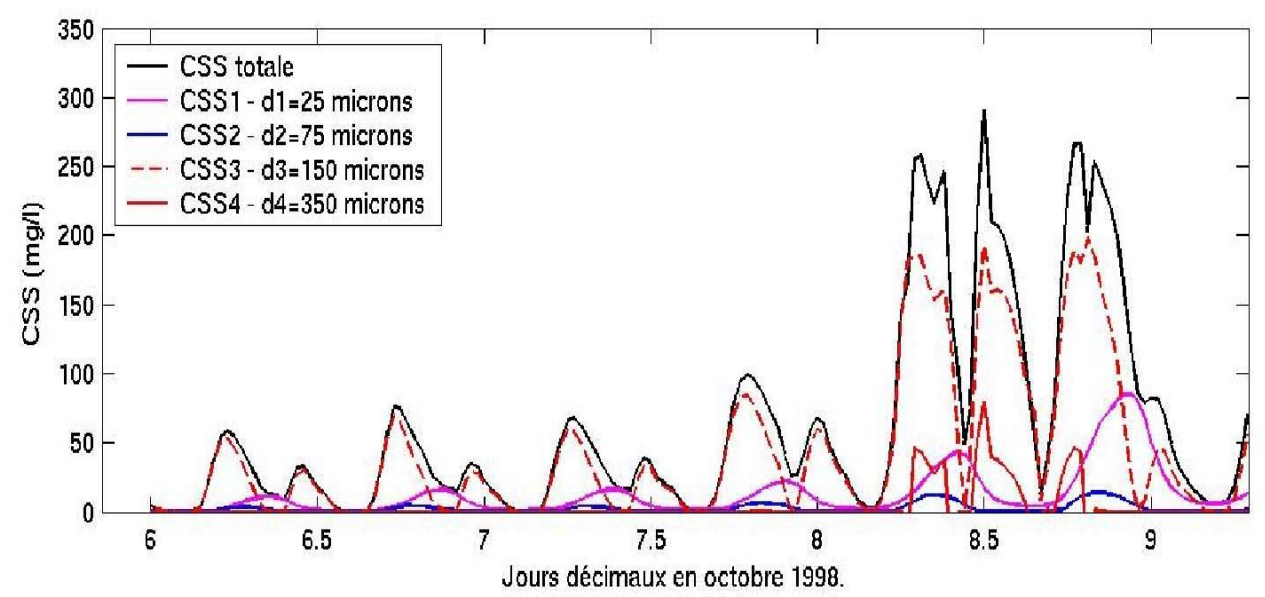

Figure 7 : Chroniques de la CSS totale et des CSS des quatre premières classes prédites à 0,3 m au-dessus du fond au site de Merlimont en octobre 1998. 


\section{Conclusions}

Ce travail illustre, à travers une comparaison des résultats du modèle avec les observations, les effets de la houle au niveau d'une zone côtière de la Manche Orientale. Les prédictions reproduisent de manière satisfaisante les observations des courants et la CSS totale effectuées à proximité du fond au large de la plage de Merlimont. Les interactions entre le courant de marée et la houle conduisent (i) en condition de houle modérée, à des modulations de la hauteur $\mathrm{h}_{\mathrm{m} 0}$ par les courants de marée et de la vitesse orbitale près $d u$ fond $u_{b, r m s}$ par l'évolution temporelle de l'élévation de la surface libre, et (ii) en période de tempête, à une augmentation d'un facteur 4 de la CSS totale dominée par la contribution des sables fins $\left(\mathrm{d}_{3}=150\right.$ $\mu \mathrm{m})$. Ces résultats, en lien avec ceux obtenus en condition de marée seule (GUILLOU \& CHAPALAIN, 2006 ; GUILLOU, 2007), contribuent à la mise en place d'un modèle de prédiction des flux de transport en suspension entre la Manche Orientale et la partie méridionale de la Mer du Nord pour différentes conditions de marée et climats de houle.

\section{Remerciements:}

Les auteurs tiennent à remercier José OZER du Management Unit of the North Sea Mathematical Models (Bruxelles, Belgique) pour la mise à disposition des harmoniques de marée extraites de sa modélisation sur la plateforme Atlantique et Michel BENOIT pour l'extraction et la mise à disposition des données de l'atlas numérique de houle.

\section{Références bibliographiques}

1 BENOIT M., LAFON F. (2004). A nearshore wave atlas along the coasts of France based on the numerical modelling of wave climate over 25 years. Coastal Engineering. pp 714-726.

2 BOOIJ N.R.C., RIS R.C., HOLTHUIJSEN L.H. (1999). A third generation wave model fro coastal regions, part i, model description and validation. Journal of Geophysical Research, 104, C4, pp 7649-7666.

3 CABIOCH L., GENTIL F., GLACON R., RETIERE C. (1977). Le macrobenthos des fonds meubles de la Manche; distribution générale et écologie. Biology of benthic organisms, Pergamon Press, Oxford, pp 115-128.

4 CHAPALAIN G., THAIS L. (2000). Tide, turbulence and suspended sediment modelling in the eastern English Channel. Coastal Engineering 41, pp 295-316. 5 CUGIER P. (2000). Développement d'un modèle numérique multicouche hétérométrique pour la simulation du transport sédimentaire en Manche : caractérisation des principaux processus. Techn. Rep. Rapport DPRE/SERNAT/2000-24, IRSN/LRC. 
6 GRANT W.D., MADSEN O.S. (1979). Combined wave and current interaction with a rough bottom. Journal of Geophysical Research, 84, C4, pp 1797-1808. 7 GROCHOWSKI N., COLLINS M. (1994). Wave activity on the sea-bed of the English Channel. Journal of Marine Biology Association U.K., 74, pp 739-742. 8 GUILLOU N. (2007). Rôles de l'hétérogénéité des sédiments de fond et des interactions houle-courant sur l'hydrodynamique et la dynamique sédimentaire en zone subtidale - applications en Manche orientale et à la pointe de la Bretagne. Thèse de doctorat de l'Université de Bretagne Occidentale, 469 p. 9 GUILlOU N., CHAPALAIN G. (2006). Modélisation des mises en suspension dans le détroit du Pas-de-Calais sous l'influence des courants de marée et de la houle. IXèmes Journées Nationales Génie Côtier-Génie Civil. 10 LARSONNEUR C., BOUYSSE P., AUFFERT J.P. (1982). The superficial sediments of the English Channel and its western approaches. Sedimentology, 29, pp 851-864.

11 LEPRETRE A., CHAPALAIN G., CARPENTIER P. (2006). Une méthode d'interpolation des caractéristiques granulométriques des sédiments superficiels. Bulletin de la Société Géologique de France, 177, 2, pp 89-95.

12 LUYTEN P.J., JONES J.E., PROCTOR R., TABOR A., TETT P., WILDADEN K. (1999). Coherens: A Coupled Hydrodynamics-Ecological model for REgioNals and Shelf seas. Management Unit of the Mathematical Models of the North Sea, Belgium.

13 SALOMON J.C., BRETON M. (1991). Courants résiduels de marée dans la Manche. Oceanologica Acta, 11, pp 47-53.

14 VASLET D., LARSONNEUR C., AUFFRET J.P. (1978). Les sédiments superficiels de la Manche au 1/500000. Carte géologique de la marge continentale française. Ministère de l'Industrie, Bureau de Recherches Géologiques et Minières, Centre National pour l'Exploitation des Océans. 15 VELEGRAKIS A.F., BISHOP C., LAFITE R., OIKONOMOU E.K., LECOUTURIER M., COLLINS M.B. (1996). Investigation of meso and macroscale sediment transport. Hydrodynamics biogeochemical processes and fluxes in the Channel, Fluxmanche II, Final Report, MAST II. MAST2CT940089, pp 128143.

16 VILLARET C., DAVIES A.G. (2004). Numerical modeling of littoral sand transport. Proceedings of the $29^{\text {th }}$ International Conference on Coastal Engineering, Lisbon, September 2004, World Scientific, Singapore, pp. 16781689. 